\title{
Normal Pupillary Response
}

National Cancer Institute

\section{Source}

National Cancer Institute. Normal Pupillary Response. NCI Thesaurus. Code C99136.

A finding indicating the quick and brisk constriction of the pupils in response to light. 